\title{
Local controllability of quantum systems
}

\author{
Zbigniew Puchała
}

Received: 20 January 2012 / Accepted: 8 March 2012 / Published online: 22 March 2012

C The Author(s) 2012. This article is published with open access at Springerlink.com

\begin{abstract}
We give a criterion that is sufficient for controllability of multipartite quantum systems. We generalize the graph infection criterion to the quantum systems that cannot be described with the use of a graph theory. We introduce the notation of hypergraphs and reformulate the infection property in this setting. The introduced criterion has a topological nature and therefore it is not connected to any particular experimental realization of quantum information processing.
\end{abstract}

Keywords Quantum control · Local control · Hypergraph

\section{Introduction}

The controllability of a given quantum system is a fundamental issue of the quantum information science. It concerns whether it is possible to drive a quantum system into a previously fixed state. There have been proposed different notations of controllability of quantum systems, such as state controllability, equivalent state controllability or operator controllability $[1,3,8]$. In the case of finite dimensional quantum systems the criteria for controllability can be expressed in terms of Lie-algebraic concepts [2,8,9]. These concepts provide a mathematical tool, in the case of closed quantum systems, i.e. systems without external influences. However, the Lie-algebraic criteria may be difficult to check, especially when the dimension of the system in question is large. For this reason there has been proposed methods for verifing controllability based on a graph theory $[6,7,15,16]$ and in many cases this setting provides an easier way for controllability inspection.

Z. Puchała $(\bowtie)$

Institute of Theoretical and Applied Informatics, Polish Academy of Sciences, Bałtycka 5,

44-100 Gliwice, Poland

e-mail: z.puchala@iitis.pl 
It is an important question whether the system is controllable when the control is performed only on a subsystem. This kind of approach is called a local-controllability and can be considered only in the case when the subsystems of a given system interact. For examples may serve coupled spin chains or spin networks [6-8].

In [7] there has been derived a graph infection criterion that ensures the controllability by relaxation and in [6] it has been shown, that it also can be used in the case of algebraic control. In this paper we generalize the graph infection criterion to the quantum systems that cannot be described with the use of a graph theory, like spin $S=1 / 2$ extended $X Y$ model $[13,14]$ or $p$-spin interaction model $[4,10]$. In order to provide new criteria for controllability, we introduce the notation of hypergraphs and reformulate the infection property in this setting. The introduced hypergraphs infection criterion has a topological nature and therefore it is not connected to any particular experimental realization of quantum information processing.

This paper is organized as follows. In Sect. 2 we provide a general description of a quantum mechanical control system. We introduce notation of controllability and provide a necessary and sufficient criteria of controllability of quantum systems. In Sect. 3 we introduce a notion of local controllability, provide a definition of hypergraph, define hypergraph infection property and finally give a new criterion for controllability. In Sect. 4 we provide the summary of the presented work and give some concluding remarks.

\section{Quantum mechanical control systems}

The dynamics of closed quantum systems can be described by a Schrödinger equation

$$
\frac{d}{d t}|\psi(t)\rangle=-i H(t)|\psi(t)\rangle
$$

where $|\psi(t)\rangle$ is an element of the complex sphere $S^{N-1}$ representing a pure state, $H(t)$ is a matrix function, which is Hermitian for every $t$ and called a Hamiltonian of the system. In this paper we assume, that the Hamiltonian is in the form $H(t)=$ $H_{\delta}+\sum_{i=1}^{M} h_{i}(t) H_{i}$, thus a quantum system can be described as

$$
\frac{d}{d t}|\psi(t)\rangle=-i\left(H_{\delta}+\sum_{i=1}^{M} h_{i}(t) H_{i}\right)|\psi(t)\rangle,
$$

where matrices $H_{\delta}, H_{1}, H_{2}, \ldots, H_{M}$ are Hermitian. Term $H_{\delta}$ is called a drift term since it drives an evolution in no control is applied. For given controls, the Eq. (2) is (time-variant) linear, and thus has unique solution. In this case the system is bilinear [9], with specialization that drift and control matrices are skew-Hermitian.

The solution of (2) can be given as

$$
|\psi(t)\rangle=U(t)|\psi(0)\rangle
$$


where $|\psi(0)\rangle$ is an initial condition and $U(t)$ is the solution of an operator equation

$$
\frac{d}{d t} U(t)=-i\left(H_{\delta}+\sum_{i=1}^{M} h_{i}(t) H_{i}\right) U(t)
$$

with an initial condition $U(0)=\mathbb{1}(N \times N$ identity matrix $)$. The solution $U(t)$ is an element of Lie group of unitary matrices $\mathrm{U}(N)$, if we assume that $\operatorname{tr} H_{\delta}=\operatorname{tr} H_{i}=0$, then the solution is in a group of special unitary matrices $\mathrm{SU}(N)$.

There are various notion of controllability for the system given in (2), [1,3,8]. Here we will consider two of them.

Definition 1 (Operator Controllable System) We call system (2) Operator Controllable if it is possible to drive an operator $X$ in (4) to any value in $\mathrm{U}(N)$ (or $\mathrm{SU}(N)$ ).

Definition 2 (State Controllable System) We call system (2) State Controllable if it is possible to drive the state $|\psi\rangle$ from the complex sphere $S^{N-1}$ to any other state on the sphere.

One can also define Equivalent State Controllable System where it is possible to drive any initial state to any element on the complex sphere modulo a phase factor, but since from a physics point of view states that differ only by a phase factor are indistinguishable, thus the equivalent state controllability is equivalent to state controllability.

The main theorem concerning controllability conditions on bilinear quantum systems follows from more general fact concerning controllability on Lie groups and was proved in the 1970's of the last century [12]. If we specify the theorem to the bilinear quantum systems it can be stated as follows.

Theorem 1 Let us denote by $\mathcal{L}$ the Lie algebra generated by the matrices $i H_{\delta}, i H_{1}$, $\ldots, i H_{M}$, i.e. $\mathcal{L}=\left\{i H_{\delta}, i H_{1}, \ldots, i H_{M}\right\}_{\mathfrak{L}}$. We have the following

- The system is operator controllable if and only if the Lie algebra $\mathcal{L}$ is an algebra $u(N)$ or $\operatorname{su}(N)$.

- The system is state controllable if and only if $\mathcal{L}$ is $u(N), s u(N)$ or in the case of even $N$ the algebra $\mathcal{L}$ is isomorphic to $\operatorname{sp}\left(\frac{N}{2}\right)^{2}$.

\section{Local controllability}

Let $V=C \cup \bar{C}$ be a given composite system, with Hamiltonian in the following form

$$
H=H_{\delta}+\sum_{k=1}^{M} h_{k}(t) H_{k},
$$

where $H_{\delta}$ is a drift-in most situations coupling Hamiltonian on whole system, and $H_{k}$ are local Hamiltonians acting on subsystem $C$, thus are in the form $H_{k}=H_{k}^{C} \otimes \mathbb{1}_{\bar{C}}$. The action of Hamiltonians $H_{k}$ are governed by time depended parameters $h_{k}(t)$. By the Theorem $1, V$ is operator controllable if and only if $i H_{\delta}$ and $i H_{k}$ are 
generators of the Lie algebra of skew-Hermitian operators on the composite system $V$, i.e. $\left\{i H_{\delta}, i H_{1}, i H_{2}, \ldots, i H_{M}\right\}_{\mathfrak{L}}=u(V)$. In this paper we assume, that the control Hamiltonians generates the full unitary algebra on a subsystem $C$, i.e. $\left\{i H_{1}^{C}, i H_{2}^{C}, \ldots, i H_{M}^{C}\right\}_{\mathfrak{L}}=u(C)$.

In article [6] was given a sufficient criterion that guarantees that a many-body quantum system with drift described by a network can be controlled by properly manipulating the (local) Hamiltonian of one of its subsystems. The criterion is based on a topological properties of the graph defined by the coupling terms in a drift Hamiltonian $H_{\delta}$. The applications of above result in the case of Heisenberg spin chains are presented in a paper [11].

\subsection{System specification}

Assume, that we have a composite system $\mathcal{X}=\mathcal{X}_{1} \cup \mathcal{X}_{2} \cup \cdots \cup \mathcal{X}_{n}$. The associated Hilbert space we denote by $\mathcal{H}^{\mathcal{X}}=\mathcal{H}^{\mathcal{X}_{1}} \otimes \cdots \otimes \mathcal{H}^{\mathcal{X}_{n}}$. Let $i$ be an identifier of a system $\mathcal{X}_{i}$ and by $X$ we denote a set of subsystems identifiers $X=\{1,2, \ldots, n\}$. The dimension of the Hilbert space $\mathcal{H}^{\mathcal{X}}$ is equal to $N=\operatorname{dim}\left(\mathcal{H}^{\mathcal{X}}\right)=\prod_{i=1}^{M} \operatorname{dim}\left(\mathcal{H}^{\mathcal{X}_{i}}\right)$.

We say, that a Hermitian operator $H$ acting on $\mathcal{H}^{\mathcal{X}}$ acts only on a subsystem $i$ if $H$ is in the form

$$
H=\mathbb{1} \otimes \cdots \otimes \mathbb{1} \otimes H_{i} \otimes \mathbb{1} \otimes \cdots \otimes \mathbb{1}
$$

where, the operator $H_{i}$ acts on a $\mathcal{H}^{\mathcal{X}_{i}}$. More generally we say, that a Hermitian operator $H$ acting on $\mathcal{H}^{\mathcal{X}}$ acts only on a subsystem $P \subset X$ if

$$
\left[H, H_{i}\right]=0,
$$

for all operators $H_{i}$ acting on subsystems $i \notin P$.

As usual we denote by $u(N)$ the Lie algebra of skew-Hermitian $N \times N$ matrices. If a composite system is specified, we will use a notation $u\left(\mathcal{X}_{i}\right)$ for Lie algebra of $N \times N$ skew-Hermitian matrices acting on $\mathcal{H}^{\mathcal{X}_{i}}$. If $i$ is an identifier of a subsystem $\mathcal{X}_{i}$, we will write $u(i)$ to denote this algebra. Similarly if $P \subset X$, by $u(P)$ we denote a Lie algebra of $N \times N$ skew-Hermitian acting on a subsystem $P$.

\subsection{Hypergraphs: definitions and properties}

In this section we give a definition of a hypergraph and provide an infection property on a hypergraph. The infection property for hypergraphs has been adopted from papers $[7,6]$, where it was defined for graphs and used to provide controllability conditions for quantum networks.

Definition 3 (Hypergraph) Let $X=\left\{x_{1}, x_{2}, \ldots, x_{n}\right\}$ be a finite set of nodes, and let $\mathcal{E}=\left(E_{1}, E_{2}, \ldots, E_{k}\right)$ be a family of subsets of $X$, we will call them edges. If we have, that $E_{i} \neq \emptyset$ for $i=1,2, \ldots, k$ and $\cup_{i=1}^{k} E_{i}=X$, then we call $(X, \mathcal{E})$ a hypergraph (see [5]). In this paper we assume, that $X=\{1,2, \ldots, n\}$. 
Fig. 1 Graphical representation of hypergraph with nodes $X=\{1,2, \ldots, 6\}$ and edges $\mathcal{E}=\left\{E_{1}, E_{2}, E_{3}\right\}=$ $\{\{1,2,4\},\{2,3,5\},\{4,5,6\}\}$

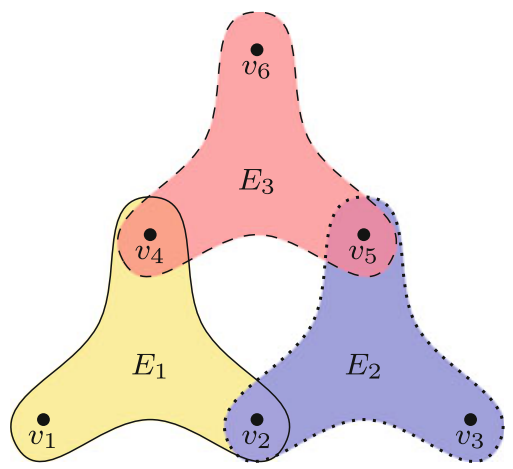

The infection process on a hypergraph can be stated as follows: if some set of nodes $A$ is infected, then the infection spreads onto a healthy neighbours $B$ if there exists edge $E$ joining some elements of infected group $A_{1} \subset A$ with $B, E=A_{1} \cup B$. Moreover $E$ is the only edge that joins elements from $A_{1}$ with healthy nodes.

Definition 4 (Infection spread) Assume that $A \subset X$ is infected, we say that infection can spread onto $B \subset X$ from $A$, where $A \cap B=\emptyset$ if

$$
\exists_{E \in \mathcal{E}} E \cap A \neq \varnothing \text { and } E \backslash A=B \text {, }
$$

moreover if $x \in(E \cap A)$ and $x \in F$ for some $F \in \mathcal{E}$, then $F=E$ or $F \subset A$.

If there exists an initial set of nodes $C$ that can infect whole hypergraph, we call such set infecting.

Definition 5 (Hypergraph infection property) For a hypergraph $(X, \mathcal{E})$ we call a subset $C \subset X$ infecting if there exist a sequence $C=P_{1} \varsubsetneqq P_{2} \varsubsetneqq \cdots \varsubsetneqq P_{m}=X$ such that an infection can spread from $P_{i}$ onto $P_{i+1} \backslash P_{i}$ for $i=1,2, \ldots, m-1$.

Example 1 To illustrate the above definitions we provide an example. Let us define a hypergraph $(X, \mathcal{E})$ with nodes $X=\{1,2, \ldots, 6\}$ and edges $\mathcal{E}=\left\{E_{1}, E_{2}, E_{3}\right\}=$ $\{\{1,2,4\},\{2,3,5\},\{4,5,6\}\}$. The graphical representation of the hypergraph is presented in Fig. 1.

If one assumes, that a set $A=\{1,2,4\}$ is infected, then it is easy to see, that the infection can spread e.g. onto $\{3,5\}$ by an edge $E_{2}=\{2,3,5\}$. Similarly if a set $\{1,2,3,4,5\}$ is infected, then infection can spread onto $\{6\}$ by an edge $E_{3}=\{4,5,6\}$. The above gives us, that the hypergraph $(X, \mathcal{E})$ has an infection property, with an infecting set $C=\{1,2,4\}$ and a sequence $C=P_{1}=\{1,2,4\} \varsubsetneqq P_{2}=\{1,2,3,4,5\} \varsubsetneqq$ $P_{3}=\{1,2,3,4,5,6\}=X$.

\subsection{Propagation property}

To provide a controllability conditions we must assume that the drift Hamiltonian meets some criteria. To do so we introduce a notion of propagating property, which relates the underlying hypergraph with appropriate Lie algebras. 
Definition 6 (Propagating property) Assume, that we have a composite system $\mathcal{X}=$ $\mathcal{X}_{1} \cup \mathcal{X}_{2} \cup \cdots \cup \mathcal{X}_{n}$. Let $(X, \mathcal{E})$ be a hypergraph. The nodes of the hypergraph are the subsystems identifiers $X=(1,2, \ldots, n)$. The edges are related to the Hermitian operator $H$, which acts on a $\mathcal{H}^{\mathcal{X}}$. The relation is given by

$$
H=\sum_{E \in \mathcal{E}} H_{E},
$$

where $H_{E}$ are Hermitian operators acting on subsystem $E$. We say, that $H$ has a propagating property if for all $E \in \mathcal{E}$, and for all $\emptyset \neq E^{\prime} \subset E$, we have

$$
\left\{\left[i H_{E}, u\left(E^{\prime}\right)\right], u\left(E^{\prime}\right)\right\}_{\mathfrak{L}}=u(E) .
$$

Example 2 Assume that we have a hypergraph $(X, \mathcal{E})$ described in Example 1. We also assume, that a composite system is composed with six qubits and for an edge $E=\left\{e_{1}, e_{2}, e_{3}\right\}$ of the hypergraph the Hermitian operator $H_{E}$ is given by

$$
i H_{E}=S_{2}^{e_{1}} S_{2}^{e_{2}} S_{2}^{e_{3}}+S_{3}^{e_{1}} S_{3}^{e_{2}} S_{3}^{e_{3}}+S_{1}^{e_{1}} S_{2}^{e_{2}} S_{3}^{e_{3}}+S_{2}^{e_{1}} S_{1}^{e_{2}} S_{0}^{e_{3}}+S_{3}^{e_{1}} S_{2}^{e_{2}} S_{0}^{e_{3}},
$$

where $S_{j}^{m}$ is $j$ th Pauli matrix on subsystem $m$, in this case

$$
S_{j}^{(m)}=\underbrace{\mathbb{1} \otimes \cdots \otimes \mathbb{1}}_{m-1} \otimes \sigma_{j} \otimes \underbrace{\mathbb{1} \otimes \cdots \otimes \mathbb{1}}_{6-m},
$$

with notation $\sigma_{0}=\mathbb{1}$. The Hamiltonian $H=\sum_{E \in \mathcal{E}} H_{E}$ has a propagation property. It is quite cumbersome task to check this analytically, but it can be done using computer algebra systems with symbolic computation and a procedure to generate a basis of a dynamical Lie algebra, see e.g. [8, Chapter 3.2.1]. Since the propagation property must be checked only on a small subsystem, this computation is fast and efficient.

\subsection{Main theorem}

Now we can state the main theorem, which express a new controllability criterion in notion of hypergraph infection and propagating property. The proof of the theorem follows the similar line of argument that the proof of the theorem in [6].

Theorem 2 Let us assume, that the drift Hamiltonian $H_{\delta}$ has a propagating property, we also assume, that a subset $C \subset X$ infects the hypergraph $(X, \mathcal{E})$. Then, the system is controllable if we perform, the control only on a subsystem $C$.

Proof First we will show by induction, that $u\left(P_{j}\right) \subset\left\{i H_{\delta}, u(C)\right\}_{\mathfrak{L}}$, where subsets $P_{j}$ are defined in the hypergraph infection property (Def. 5). The first induction step is obvious, since $u\left(P_{1}\right)=u(C) \subset\left\{i H_{\delta}, u(C)\right\}_{\mathfrak{L}}$. Next, we assume, that for some $j<m$, we have

$$
u\left(P_{j}\right) \subset\left\{i H_{\delta}, u(C)\right\}_{\mathfrak{L}} .
$$


Since, we have, that infection spreads from $P_{j}$ onto $P_{j+1} \backslash P_{j}$, there exist an edge $E \in \mathcal{E}$, such that $E \cap P_{j} \neq \emptyset$ and $E \backslash P_{j}=P_{j+1} \backslash P_{j}$.

We define $E^{(j)}=E \cap P_{j}$ and write

$$
\begin{aligned}
{\left[i H_{\delta}, u\left(E^{(j)}\right)\right] } & =\sum_{F \in \mathcal{E}}\left[i H_{F}, u\left(E^{(j)}\right)\right] \\
& =\left[i H_{E}, u\left(E^{(j)}\right)\right]+\sum_{F \in \mathcal{E}, F \neq E}\left[i H_{F}, u\left(E^{(j)}\right)\right] .
\end{aligned}
$$

Now using, the infection property of the graph $(X, \mathcal{E})$, we have, that the last sum above can be restricted to $F \in \mathcal{E}, F \subset P_{j}$. Thus we have

$$
\left[i H_{E}, u\left(E^{(j)}\right)\right]=\left[i H_{\delta}, u\left(E^{(j)}\right)\right]-\sum_{F \in \mathcal{E}, F \subset P_{j}}\left[i H_{F}, u\left(E^{(j)}\right)\right] .
$$

The last sum is an element of $u\left(P_{j}\right)$ and the first term of the right hand side is an element of $\left\{i H_{\delta}, u(C)\right\}_{\mathfrak{L}}$, so we obtain, that $\left[i H_{E}, u\left(E^{(j)}\right)\right] \in\left\{i H_{\delta}, u(C)\right\}_{\mathfrak{L}}$.

Now using propagation property of the drift Hamiltonian, we have

$$
\left\{\left[i H_{E}, u\left(E^{(j)}\right)\right], u\left(E^{(j)}\right)\right\}_{\mathfrak{L}}=u(E) .
$$

Since $\left\{u\left(P_{j}\right), u(E)\right\}_{\mathfrak{L}}=u\left(P_{j+1}\right)$, thus $u\left(P_{j+1}\right) \in\left\{i H_{\delta}, u(C)\right\}_{\mathfrak{L}}$.

At the last step of induction procedure $j=k$, we obtain

$$
u\left(P_{k}\right)=u(X) \subset\left\{i H_{\delta}, u(C)\right\}_{\mathfrak{L}},
$$

and since $u(X)$ is the maximal algebra which can be obtained, we have

$$
u\left(P_{k}\right)=u(X)=\left\{i H_{\delta}, u(C)\right\}_{\mathfrak{L}} .
$$

Now by Theorem 1, we obtain that the system is operator controllable.

To use the above theorem to check whether the system is controllable one first must test the propagation property for a drift Hamiltonian and ensure that the underlying hypergraph has an infection property. The second task is purely topological and can be done rather easily, while the first one-the propagation property must be checked only on a small subsystem, which reduces the complexity.

Using Theorem 2 we obtain that the dynamical system described in Examples 1, 2 is controllable by performing the control only on a subsystem $\{1,2,4\}$. We remind here the assumption, which was made at the beginning of Sect. 3, that local control Hamiltonians generates the full unitary algebra on a specified subsystem.

\section{Concluding remarks}

In this paper we provided a new controllability criterion of multipartite quantum systems based on notion of hypergraph. We generalized the graph infection criterion 
to the quantum systems that cannot be described with the use of a graph theory. To do so we have introduced a notion of local controllability, provided a definition of hypergraph, defined hypergraph infection property and finally gave a new criterion for controllability. The introduced criterion has a topological nature and therefore it is not connected to any particular experimental realization of quantum information processing.

Acknowledgments We acknowledge the financial support by the Polish National Science Centre under the grant number N N514 513340.

Open Access This article is distributed under the terms of the Creative Commons Attribution License which permits any use, distribution, and reproduction in any medium, provided the original author(s) and the source are credited.

\section{References}

1. Albertini, F., D'Alessandro, D.: Notions of controllability for quantum mechanical systems. In: Decision and control. Proceedings of the 40th IEEE conference on, vol. 2, pp. 1589-1594. IEEE (2001)

2. Albertini, F., D'Alessandro, D.: The Lie algebra structure and controllability of spin systems. Linear Algebra Appl. 350(1-3), 213-235 (2002)

3. Albertini, F., D'Alessandro, D.: Notions of controllability for bilinear multilevel quantum systems. Autom. Control, IEEE Trans. 48(8), 1399-1403 (2003)

4. Baxter, R., Wu, F.: Exact solution of an ising model with three-spin interactions on a triangular lattice. Phys. Rev. Lett. 31(21), 1294-1297 (1973)

5. Berge, C.: Graphs and Hypergraphs, vol. 6. Elsevier, North Holland, NY (1976)

6. Burgarth, D., Bose, S., Bruder, C., Giovannetti, V.: Local controllability of quantum networks. Phys. Rev. A 79(6), 60-305 (2009)

7. Burgarth, D., Giovannetti, V.: Full control by locally induced relaxation. Phys. Rev. Lett. 99(10), 100501 (2007)

8. D'Alessandro, D.: Introduction to Quantum Control and Dynamics. Chapman \& Hall, London (2008)

9. Elliott, D.: Bilinear Control Systems: Matrices in Action. Springer, Berlin (2009)

10. Goldschmidt, Y.et al. : Solvable model of the quantum spin glass in a transverse field. Phys. Rev. B Condens. Matter 41(7), 4858 (1990)

11. Heule, R., Bruder, C., Burgarth, D., Stojanovió, V.M.: Local quantum control of Heisenberg spin chains. Phys. Rev. A 82(5), 052-333 (2010). doi:10.1103/PhysRevA.82.052333

12. Jurdjevic, V., Sussmann, H.: Control systems on lie groups. J. Diff. Equ. 12(2), 313-329 (1972)

13. Lou, P.: Thermal and spin transports in spin- $1 / 2$ extended $x y$ chain. Phys. Status Solidi (B) 241(6), 1343-1349 (2004)

14. Titvinidze, I., Japaridze, G.: Phase diagram of the spin extended model. Eur. Phys. J. B-Condens. Matter Complex Syst. 32(3), 383-393 (2003)

15. Turinici, G., Rabitz, H.: Quantum wavefunction controllability. Chem. Phys. 267(1-3), 1-9 (2001)

16. Turinici, G., Rabitz, H.: Wavefunction controllability for finite-dimensional bilinear quantum systems. J. Phys. A Math. Gen. 36, 2565 (2003) 